TITLE:

\title{
Anisotropic Vector Play Model Incorporating Decomposed Shape
} Functions

\section{$\operatorname{AUTHOR}(\mathrm{S})$ :}

Fujinaga, Takashi; Mifune, Takeshi; Matsuo, Tetsuji

\section{CITATION:}

Fujinaga, Takashi ... [et al]. Anisotropic Vector Play Model Incorporating Decomposed Shape Functions. IEEE Transactions on Magnetics 2016, 52(3): 7300604.

\section{ISSUE DATE:}

2016-03

URL:

http://hdl.handle.net/2433/217060

\section{RIGHT:}

(c) 2015 IEEE. Personal use of this material is permitted. Permission from IEEE must be obtained for all other uses, in any current or future media, including reprinting/republishing this material for advertising or promotional purposes, creating new collective works, for resale or redistribution to servers or lists, or reuse of any copyrighted component of this work in other works. 


\title{
Anisotropic Vector Play Model Incorporating Decomposed Shape Functions
}

\author{
Takashi Fujinaga; Takeshi Mifune, Member, IEEE; and Tetsuji Matsuo, Member, IEEE \\ Graduate School of Engineering, Kyoto University, Kyoto 615-8510, Japan; takashi_3210@fem.kuee.kyoto-u.ac.jp
}

\begin{abstract}
We propose an accurate anisotropic vector play model that uses the decomposition of vector shape functions. The parallel and perpendicular components of the magnetic field $H$ to the magnetic flux density $B$ are independently identified in constructing the decomposed shape functions. We further propose a method to reconstruct the perpendicular component from the one-dimensional measurement of the parallel component based on a magnetic energy approach. This method accurately reconstructs the vector hysteretic properties of silicon steel under alternating and rotating magnetic flux conditions.
\end{abstract}

Index Terms - Anisotropy, magnetic energy, play model, vector hysteresis.

\section{INTRODUCTION}

A DVANCED magnetic field analyses of three-phase electric machinery require precise descriptions of the vector hysteretic property of the magnetic field at junctions of magnetic flux. The vector play model [1]-[4] is one of the most accurate and efficient models in representing the vector hysteretic property of silicon steel sheets.

Using the isotropic vector play model and the anisotropy matrix, Ref. [4] describes a successful approximation to the anisotropic vector hysteresis of non-oriented silicon steel sheets including the elliptic rotational field. In representing the dynamic vector hysteretic property, the model provides a base model of the dynamic model [5] or it is applied to the finite element eddy-current analysis [6]. However, because of the simple construction of the anisotropy matrix, the representation may not be highly accurate when the anisotropy becomes strong. Another drawback of this model is the need to measure the alternating magnetic property using a 2D or rotational single sheet tester (SST) [7], where precise waveform control is needed to maintain the B-vector along its specified direction.

For an accurate anisotropy representation, this paper proposes anisotropic shape functions that represent the perpendicular component of the magnetic field $\boldsymbol{H}$ to the magnetic flux density $\boldsymbol{B}$ independently of its parallel component. Based on a magnetic energy approach [8], [9], we further propose a method to reconstruct the perpendicular component without its direct measurement using only the 1-D measurement of the parallel component.

\section{Vector Play Model}

From the magnetic flux density $\boldsymbol{B}$ as input, the anisotropic vector play model provides the magnetic field $\boldsymbol{H}$ as output:

Manuscript received April 1, 2015; revised May 15, 2015 and June 1, 2015; accepted July 1, 2015. Date of publication July 10, 2015; date of current version July 31, 2015. Corresponding author: T. Matsuo (e-mail: matsuo.tetsuji.5u@kyoto-u.ac.jp).

Color versions of one or more of the figures in this paper are available online at http://ieeexplore.ieee.org.

Digital Object Identifier (inserted by IEEE).

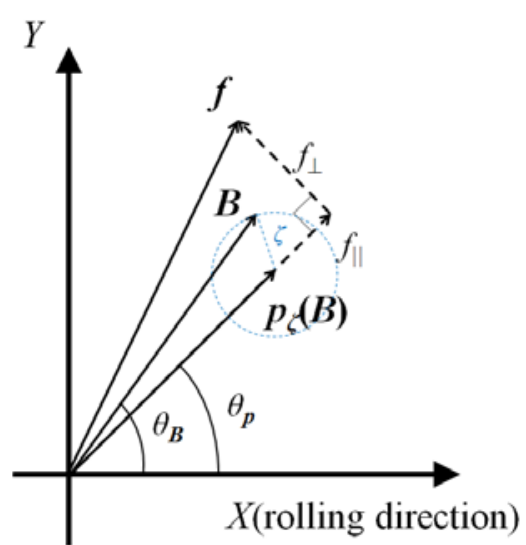

Fig.1. Vector play hysteron and vector shape function.

$$
\begin{gathered}
\boldsymbol{H}=\boldsymbol{P}(\boldsymbol{B})=\int_{0}^{B_{S}} \boldsymbol{f}\left(\zeta, \boldsymbol{p}_{\zeta}(\boldsymbol{B}), \boldsymbol{B}\right) \mathrm{d} \zeta, \\
\boldsymbol{p}_{\zeta}(\boldsymbol{B})=\boldsymbol{B}-\frac{\zeta\left(\boldsymbol{B}-\boldsymbol{p}_{\zeta}^{0}\right)}{\max \left(\zeta,\left|\boldsymbol{B}-\boldsymbol{p}_{\zeta}^{0}\right|\right)},
\end{gathered}
$$

where $\boldsymbol{f}$ is the anisotropic vector shape function, $\boldsymbol{p}_{\zeta}$ is the vector play hysteron having radius $\zeta, \boldsymbol{p}_{\zeta}^{0}$ is the vector $\boldsymbol{p}_{\zeta}$ at the previous time-point, and $B_{\mathrm{S}}$ is the saturation magnetic flux density. Ref. [4] proposed a simple form for the shape function,

$$
\boldsymbol{f}\left(\zeta, \boldsymbol{p}_{\zeta}(\boldsymbol{B}), \boldsymbol{B}\right)=\boldsymbol{W}\left(|\boldsymbol{B}|, \theta_{B}\right) \boldsymbol{f}_{0}\left(\zeta, \boldsymbol{p}_{\zeta}(\boldsymbol{B})\right),
$$

where $\boldsymbol{W}$ is the anisotropy matrix, $\theta_{B}$ the azimuth angle of $\boldsymbol{B}$, and $\boldsymbol{f}_{0}$ the isotropic vector shape function.

We propose two more accurate representations of the vector shape function,

$$
\boldsymbol{f}\left(\zeta, \boldsymbol{p}_{\zeta}(\boldsymbol{B}), \boldsymbol{B}\right)=f_{\|}\left(\zeta, \mid \boldsymbol{p}_{\zeta}(\boldsymbol{B}), \theta_{B}\right) \boldsymbol{e}_{p_{\|}}+f_{\perp}\left(\zeta,\left|\boldsymbol{p}_{\zeta}(\boldsymbol{B})\right| \theta_{B}\right) \boldsymbol{e}_{p_{\perp}}
$$

and

$$
\boldsymbol{f}\left(\zeta, \boldsymbol{p}_{\zeta}(\boldsymbol{B})\right)=f_{\|}\left(\zeta, \boldsymbol{p}_{\zeta}(\boldsymbol{B}), \theta_{p}\right) \boldsymbol{e}_{p_{\|}}+f_{\perp}\left(\zeta,\left|\boldsymbol{p}_{\zeta}(\boldsymbol{B})\right|, \theta_{p}\right) \boldsymbol{e}_{p_{\perp}}
$$

where $\theta_{p}$ is the azimuth angle of $\boldsymbol{p}_{\zeta}, f_{\|}$and $f_{\perp}$ are the shape functions for the parallel and perpendicular component of $\boldsymbol{H}$ to $\boldsymbol{p}_{\zeta}$, and $\boldsymbol{e}_{\mathrm{p} \|}$ and $\boldsymbol{e}_{\mathrm{p} \perp}$ denote the parallel and perpendicular unit vectors to $\boldsymbol{p}_{\zeta}$ (see Fig. 1). For the anisotropy representation, the shape functions, (4) and (5), depend on the azimuth angles of $\boldsymbol{B}$ and $\boldsymbol{p}_{\zeta}$, respectively. 


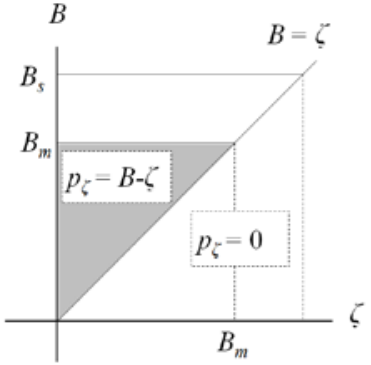

(a) normal magnetization curve

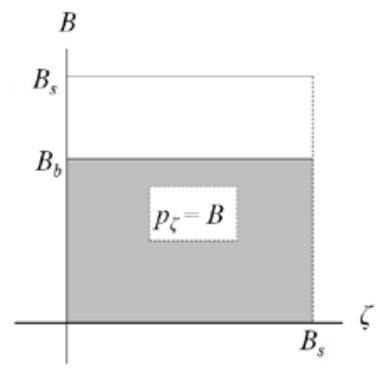

(b) anhysteretic curve
Fig. 2. Integral domains for the normal magnetization and anhysteretic curves

The shape functions $f_{\|}$and $f_{\perp}$ are determined from the measured parallel and perpendicular components of $\boldsymbol{H}=\left(H_{\|}(B\right.$, $\left.\left.\theta_{B}\right), H_{\perp}\left(B, \theta_{B}\right)\right)$ using the identification method for a scalar play model [10].

\section{MAGNETIC ENERGY APPROACH}

\section{A. Magnetic energy and 2-D magnetic property}

The vector components of $\left(H_{\|}\left(B, \theta_{B}\right), H_{\perp}\left(B, \theta_{B}\right)\right)$ are measured under the alternating flux condition along the $\theta_{B^{-}}$ direction. The measurement of $H_{\perp}(B)$ requires the 2-D SST, where a sophisticated waveform control is required to maintain $\boldsymbol{B}$ along its specified direction. In contrast, $H_{\|}\left(B, \theta_{B}\right)$ can be measured by the conventional 1-D SST or the Epstein frame using strip samples cut along the $\theta_{B}$ directions.

Refs. [8] and [9] proposed a useful method to determine $H_{\perp}\left(B, \theta_{B}\right)$ without its measurement using the magnetic energy. The magnetic energy density $F$ is defined as

$$
F\left(B, \theta_{B}\right)=\int_{0}^{B} \boldsymbol{H} \cdot d \boldsymbol{B}=\int_{0}^{B} H_{\|}\left(B, \theta_{B}\right) \mathrm{d} B .
$$

It is assumed that $\boldsymbol{H}$ and $\boldsymbol{B}$ have no hysteretic relation and that the integration is independent of the path. Using the magnetic energy (6), the components of $\boldsymbol{H}$ are given as

$$
H_{\|}=\frac{\partial F}{\partial B}, H_{\perp}=\frac{1}{B} \frac{\partial F}{\partial \theta_{B}} .
$$

As (7) is derived without hysteresis, it cannot be used directly to determine $f_{\perp}$ but can be obtain with a method developed below via two magnetization curves without hysteresis, the normal magnetization curve and the anhysteretic curve, to be applied in (6) and (7)

\section{B. Derivation from normal magnetization curve}

The normal magnetization curve is obtained by the measurement of symmetric $B-H$ loops under the alternating magnetic flux condition. The play model does not distinguish the normal magnetization curve from the initial magnetization curve. For the alternating input of amplitude $B_{\mathrm{m}}$ along the $\theta_{\mathrm{B}}$ direction, the input $\boldsymbol{B}=B_{\mathrm{m}} \boldsymbol{e}_{\mathrm{B} \|}$ gives

$$
\boldsymbol{p}_{\zeta}\left(B_{\mathrm{m}} \boldsymbol{e}_{\mathrm{B} \|}\right)=\left\{\begin{array}{cc}
\left(B_{\mathrm{m}}-\zeta\right) \boldsymbol{e}_{\mathrm{B} \|} & \left(\zeta<B_{\mathrm{m}}\right), \\
0 & \left(B_{\mathrm{m}} \leq \zeta\right)
\end{array}\right.
$$

where $\boldsymbol{e}_{\mathrm{B} \|}$ is the unit vector parallel to $\boldsymbol{B}$. Assuming $f_{\|}(\zeta, 0, \theta)=$ $f_{\perp}(\zeta, 0, \theta)=0, \boldsymbol{H}_{\mathrm{m}}\left(B_{\mathrm{m}} \boldsymbol{e}_{\mathrm{B} \|}\right)=\left(H_{\mathrm{m} \|}, H_{\mathrm{m} \perp}\right)$ is given by

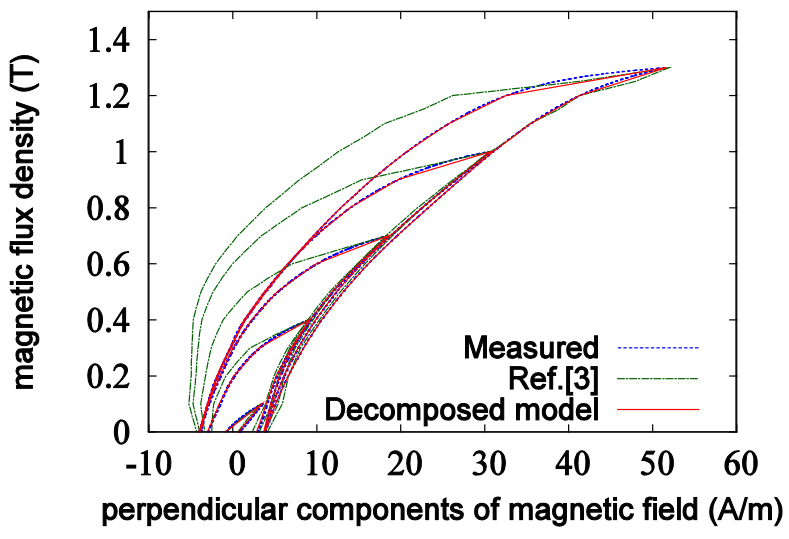

Fig. 3. Simulated $B-H_{\perp}$ loops for $\theta_{B}=\pi / 6$ using the shape functions (3) and (4), where $f_{\perp}$ in (4) is identified from measured data.

$$
\begin{gathered}
H_{m \|}\left(B_{m}, \theta_{B}\right)=\int_{0}^{B_{m}} f_{\|}\left(\zeta, B_{m}-\zeta, \theta_{B}\right) \mathrm{d} \zeta, \\
H_{m \perp}\left(B_{m}, \theta_{B}\right)=\int_{0}^{B_{m}} f_{\perp}\left(\zeta, B_{m}-\zeta, \theta_{B}\right) \mathrm{d} \zeta .
\end{gathered}
$$

From (6) and (9), the magnetic energy density $F$ is represented as

$$
\begin{aligned}
F\left(B_{m}, \theta_{B}\right) & =\int_{0}^{B_{m}} H_{m \|}\left(B, \theta_{\mathrm{B}}\right) \mathrm{d} B \\
& =\int_{0}^{B_{m}}\left\{\int_{0}^{B} f_{\|}\left(\zeta, B-\zeta, \theta_{B}\right) \mathrm{d} \zeta\right\} \mathrm{d} B, \\
& =\int_{0}^{B_{m}}\left\{\int_{\zeta}^{B_{m}} f_{\|}\left(\zeta, B-\zeta, \theta_{B}\right) \mathrm{d} B\right\} \mathrm{d} \zeta
\end{aligned}
$$

where the integral domain is shown in Fig. 2(a). From (7) and (11), $H_{m \perp}$ is given as

$$
H_{m \perp}\left(B_{m}, \theta_{B}\right)=\int_{0}^{B_{m}} \frac{1}{B_{m}} \frac{\partial}{\partial \theta_{B}}\left\{\int_{\zeta}^{B_{m}} f_{\|}\left(\zeta, B-\zeta, \theta_{B}\right) \mathrm{d} B\right\} \mathrm{d} \zeta
$$

From a comparison of (10) with (12), $f_{\perp}$ is estimated as

$$
f_{\perp}\left(\zeta, B_{m}-\zeta, \theta_{B}\right)=\frac{1}{B_{m}} \frac{\partial}{\partial \theta_{B}}\left\{\int_{\zeta}^{B_{m}} f_{\|}\left(\zeta, B-\zeta, \theta_{B}\right) \mathrm{d} B\right\} .
$$

Equation (13) is rewritten as

$$
f_{\perp}\left(\zeta, B, \theta_{B}\right)=\frac{1}{B+\zeta} \frac{\partial}{\partial \theta_{B}}\left\{\int_{0}^{B} f_{\|}\left(\zeta, B, \theta_{B}\right) \mathrm{d} B\right\} .
$$

\section{Derivation from anhysteretic curve}

The anhysteretic curve is obtained by applying the decaying alternating magnetic flux with biased direct magnetic flux density $B_{\mathrm{b}}$. The resultant $\boldsymbol{p}_{\zeta}\left(B_{\mathrm{b}} \boldsymbol{e}_{\mathrm{B} \|}\right)$ is given by

$$
\boldsymbol{p}_{\zeta}\left(B_{\mathrm{b}} \boldsymbol{e}_{\mathrm{B} \|}\right)=B_{\mathrm{b}} \boldsymbol{e}_{\mathrm{B} \|} \text {. }
$$

Consequently, $\boldsymbol{H}_{\mathrm{b}}\left(B_{\mathrm{b}} \boldsymbol{e}_{\mathrm{B} \|}\right)=\left(H_{\mathrm{b} \|}, H_{\mathrm{b} \perp}\right)$ is written as:

$$
\begin{aligned}
& H_{b \|}\left(B_{b}, \theta_{B}\right)=\int_{0}^{B_{S}} f_{\|}\left(\zeta, B_{b}, \theta_{B}\right) \mathrm{d} \zeta \\
& H_{b \perp}\left(B_{b}, \theta_{B}\right)=\int_{0}^{B_{S}} f_{\perp}\left(\zeta, B_{b}, \theta_{B}\right) \mathrm{d} \zeta .
\end{aligned}
$$

From (6) and (16), $F$ is written as 


$$
\begin{aligned}
F\left(B_{b}, \theta_{B}\right) & =\int_{0}^{B_{m}} H_{b \|}\left(B, \theta_{\mathrm{B}}\right) \mathrm{d} B \\
& =\int_{0}^{B_{b}}\left\{\int_{0}^{B_{S}} f_{\|}\left(\zeta, B, \theta_{B}\right) \mathrm{d} \zeta\right\} \mathrm{d} B ; \\
& =\int_{0}^{B_{S}}\left\{\int_{0}^{B_{b}} f_{\|}\left(\zeta, B, \theta_{B}\right) \mathrm{d} B\right\} \mathrm{d} \zeta
\end{aligned}
$$

the domain of integration is shown in Fig. 2(b). From (7) and (18), $H_{b \perp}$ is given as

$$
H_{b \perp}\left(B_{b}, \theta_{B}\right)=\int_{0}^{B_{S}} \frac{1}{B_{b}} \frac{\partial}{\partial \theta_{B}}\left\{\int_{0}^{B_{b}} f_{\|}\left(\zeta, B, \theta_{B}\right) \mathrm{d} B\right\} \mathrm{d} \zeta .
$$

By comparing (17) with (19), $f_{\perp}$ is estimated from

$$
f_{\perp}\left(\zeta, B, \theta_{B}\right)=\frac{1}{B} \frac{\partial}{\partial \theta_{B}}\left\{\int_{0}^{B} f_{\|}\left(\zeta, B, \theta_{B}\right) \mathrm{d} B\right\} .
$$

\section{Simulation Results}

We now compare simulation results of the anisotropic vector models with the measured static magnetic property for the non-oriented silicon steel sheet JIS: 35A230 under the alternating and rotational magnetic flux condition.

\section{A. Alternating magnetic flux condition}

Fig. 3 shows simulated $B-H_{\perp}$ loops using the shape function (4) for alternating flux with $\theta_{B}=\pi / 6$, where $f_{\perp}$ in (4) is identified from the measured $H_{\perp}$. The shape function (5) is equivalent to (4) for the alternating input because $\theta_{p}=\theta_{B}$. Fig. 4 shows simulated $B-H_{\perp}$ loops with $\theta_{B}=\pi / 6$ and $\pi / 3$ using $f_{\perp}$ determined from (14) and (20) without using the measured $B$ $H_{\perp}$ loops.

The proposed vector model using (4) represents $B-H_{\perp}$ loops more accurately than the simple model using (3). The shape function $f_{\perp}$ identified from the measured $H_{\perp}$ gives very accurate $B-H_{\perp}$ loops. The shape function $f_{\perp}$ determined from (20) obtains more accurate $B-H_{\perp}$ loops than $f_{\perp}$ determined from (14). Fig. 4 implies that the 2-D anisotropic vector hysteretic property can be reconstructed from the $B-H$ loops measured with the 1-D SST using the proposed anisotropic vector play model and the magnetic energy approach.

Fig. 5 shows the simulated iron loss per cycle for the alternating input with $\theta_{B}=0, \pi / 6, \pi / 4, \pi / 3$. This simulated loss agrees with the measured loss.

\section{B. Rotating magnetic flux condition}

Figs. 6 and 7 portray the simulated loci of $\boldsymbol{H}$ for the clockwise (CW) rotational input of $\boldsymbol{B}$ where the amplitude $B_{a}$ $=0.7,1.0,1.2,1.3,1.4 \mathrm{~T}$; the vector shape functions (4) and (5) are used with the shape function $f_{\perp}$ determined from (20). The loci of $\boldsymbol{H}$ for rotational inputs of $\boldsymbol{B}$ are reconstructed from the $B-H$ loops measured with the 1-D SST. The vector shape function (5) is more accurate than (4) when the amplitude of $\boldsymbol{B}$ is small.

If the measured rotational hysteresis loss is available, it is possible to adjust the simulated rotational hysteresis loss to the measured value by decomposing the vector shape function (5),

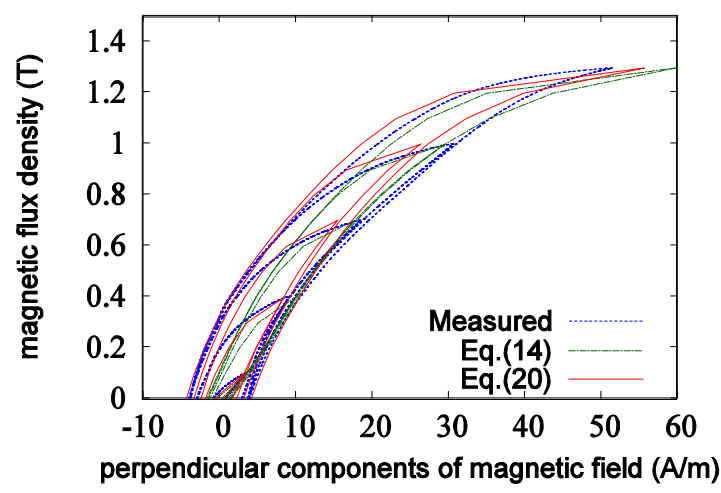

(a) $\theta_{B}=\pi / 6[\mathrm{rad}]$

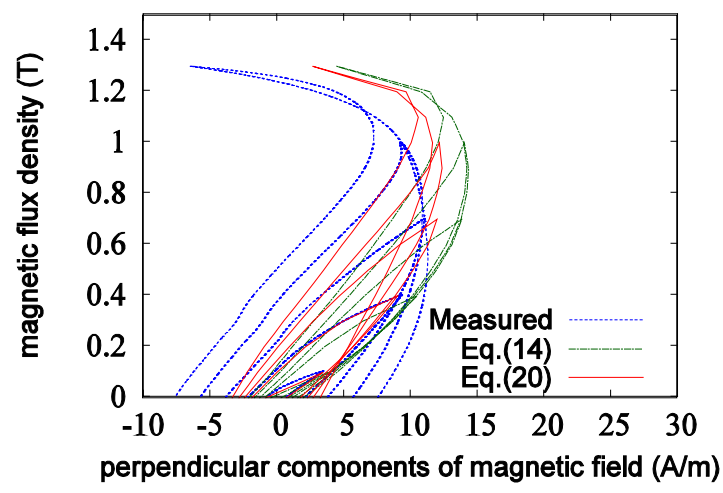

(b) $\theta_{B}=\pi / 3[\mathrm{rad}]$

Fig. 4. Simulated $B-H_{\perp}$ loops using the shape function $f_{\perp}$ determined from magnetic energy using (14) and (20).

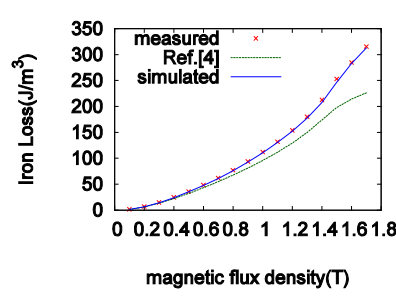

(a) $\theta_{B}=0[\mathrm{rad}]$

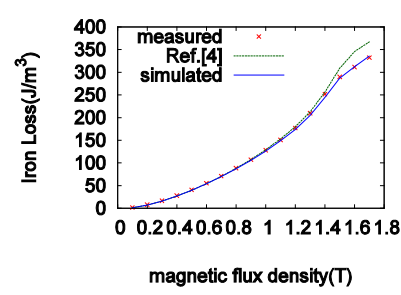

(c) $\theta_{B}=\pi / 4[\mathrm{rad}]$

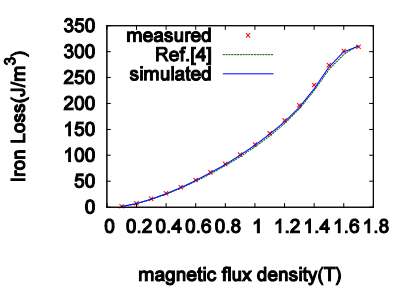

(b) $\theta_{B}=\pi / 6[\mathrm{rad}]$

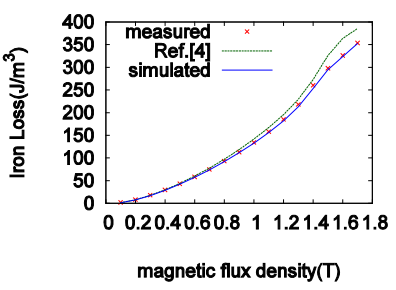

(d) $\theta_{B}=\pi / 3[\mathrm{rad}]$
Fig. 5. Alternating hysteresis loss

$$
\begin{aligned}
\boldsymbol{f}\left(\zeta, \boldsymbol{p}_{\zeta}(\boldsymbol{B}), \theta_{p}\right) & =\boldsymbol{f}_{\|\| \|}+\boldsymbol{f}_{\| \perp \perp}+\boldsymbol{f}_{\perp \perp \|}+\boldsymbol{f}_{\perp_{-} \perp} \\
\boldsymbol{f}_{\left\|\_\right\|} & =\left\{f_{\|}\left(\zeta, \mid \boldsymbol{p}_{\zeta}(\boldsymbol{B}), \theta_{p}\right) \boldsymbol{e}_{p_{\|}} \cdot \boldsymbol{e}_{B_{\|}}\right\} \boldsymbol{e}_{B_{\|}} \\
\boldsymbol{f}_{\| \perp \perp} & =\left\{f_{\|}\left(\zeta, \mid \boldsymbol{p}_{\zeta}(\boldsymbol{B}), \theta_{p}\right) \boldsymbol{e}_{p_{\|}} \cdot \boldsymbol{e}_{B_{\perp}}\right\} \boldsymbol{e}_{B_{\perp}} \\
\boldsymbol{f}_{\perp \_\|} & =\left\{f_{\perp}\left(\zeta, \mid \boldsymbol{p}_{\zeta}(\boldsymbol{B}), \theta_{p}\right) \boldsymbol{e}_{p_{\perp}} \cdot \boldsymbol{e}_{B_{B_{\|}}} \boldsymbol{e}_{B_{\|}}\right. \\
\boldsymbol{f}_{\perp \perp \perp} & =\left\{f_{\perp}\left(\zeta, \mid \boldsymbol{p}_{\zeta}(\boldsymbol{B}), \theta_{p}\right) \boldsymbol{e}_{p_{\perp}} \cdot \boldsymbol{e}_{B_{\perp}}\right\} \boldsymbol{e}_{B_{\perp}}
\end{aligned}
$$



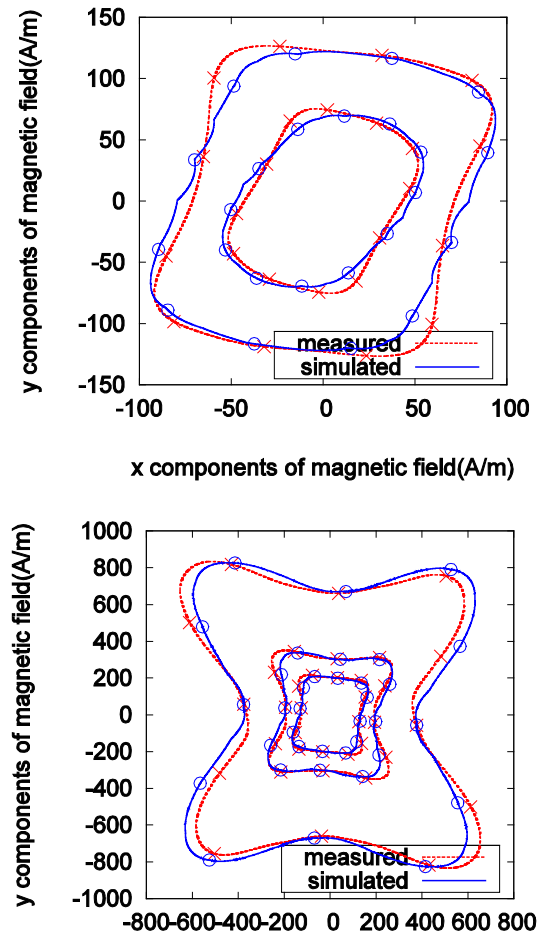

$x$ components of magnetic field $(\mathrm{A} / \mathrm{m})$

Fig. 6. Loci of $\boldsymbol{H}$ vector for rotational magnetic flux using (4).

where $\boldsymbol{e}_{\mathrm{B} \perp}$ is the unit vector perpendicular to $\boldsymbol{B}$. The alternating property depends on $\boldsymbol{f}_{\|\llcorner\|\|}$and $\boldsymbol{f}_{\perp \_\perp}$ whereas $\boldsymbol{f}_{\| \_\perp}$ and $\boldsymbol{f}_{\perp \_\|}$do not appear for the alternating input because $\boldsymbol{e}_{p \|} \cdot \boldsymbol{e}_{\mathrm{B} \perp}=\boldsymbol{e}_{p \perp} \cdot \boldsymbol{e}_{\mathrm{B} \|}=0$. The rotational hysteresis loss is affected by $\boldsymbol{f}_{\| \_\perp}$ and $\boldsymbol{f}_{\perp \_} \perp$. To adjust the rotational loss without affecting its alternating property, $\boldsymbol{f}_{\| \_\perp}$ is modified to

$$
\boldsymbol{f}_{\| \_\perp}^{*}=\left(\frac{L_{\text {mea }}-L_{\perp_{\perp}}(|\boldsymbol{B}|)}{L_{\| \_\perp}(|\boldsymbol{B}|)}\right) \boldsymbol{f}_{\| \_\perp},
$$

where $L_{\perp \_\perp}$ and $L_{\| \_\perp}$ are simulated losses associated with $\boldsymbol{f}_{\| \_\perp}$ and $\boldsymbol{f}_{\perp \_} \perp$, and $L_{\text {mea }}$ is the measured loss. This adjustment requires the measurement under the rotating magnetic flux condition using the 2D-SST, where the waveform control is relatively easy compared with the measurement under the alternating magnetic flux condition.

Fig. 8 shows the adjusted simulated iron loss per cycle of JIS:35A230 for the rotational input, which agrees with the measured loss.

\section{CONCLUSION}

The 2-D anisotropic vector hysteretic property can be reconstructed from the $B-H$ loops measured with the 1-D SST, using the proposed anisotropic vector play model and the magnetic energy approach.

\section{REFERENCES}

[1] M. A. Krasnosel'skii, A.Pokrovskii, Systems with Hysteresis, SpringerVerlag, 1983.

[2] A. Bergqvist, "Magnetic vector hysteresis model with dry friction-like pinning," Physica B, vol. 233, pp. 342-347, Jun. 1997.

[3] C. Serpico, M. d'Aquino, C. Visone, and D. Davino, "A new class of
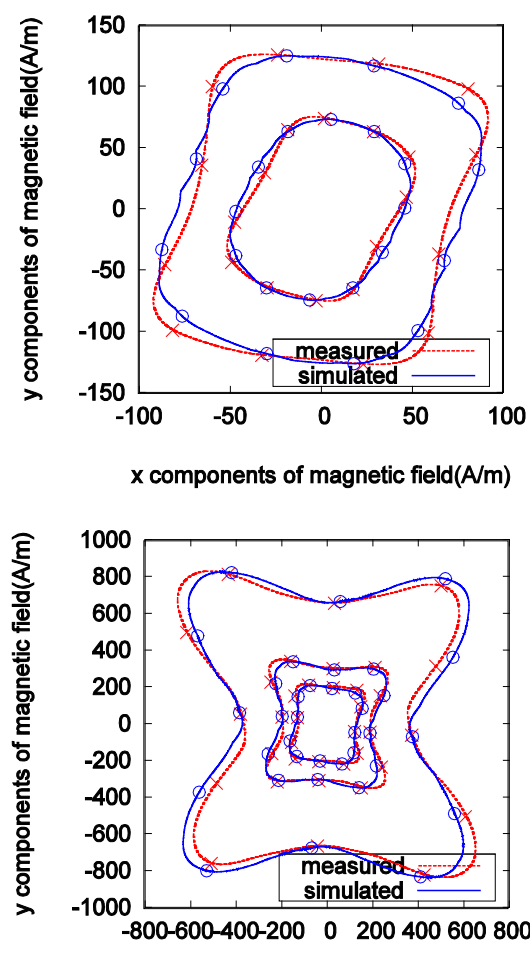

$x$ components of magnetic field $(\mathrm{A} / \mathrm{m})$

Fig. 7. Loci of $\boldsymbol{H}$ vector for rotational magnetic flux using (5).

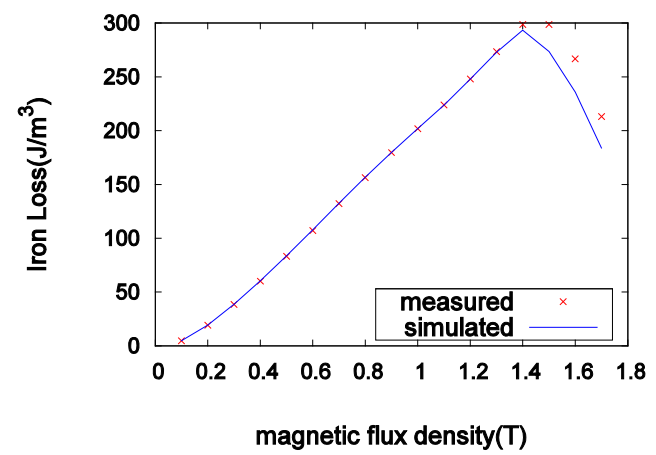

Fig. 8. Rotational hysteresis loss using (5).

Preisach-type isotropic vector model of hysteresis," Physica B, vol. 343, pp. 117-120, Jan. 2004

[4] T. Matsuo, "Anisotropic vector hysteresis model using an isotropic vector play model,” IEEE Trans. Magn., vol.46, pp. 3041-3044, Aug. 2010.

[5] T. Matsuo and M. Miyamoto, "Dynamic and anisotropic vector hysteresis model based on isotropic vector play model for non-oriented silicon steel sheet,” IEEE Trans. Magn., vol. 48, pp. 215-218, Feb. 2012.

[6] Y. Takeda, Y. Takahashi, K. Fujiwara, A. Ahagon and T. Matsuo, "Iron loss estimation method for rotating machines taking account of hysteretic property,” IEEE Trans. Magn., vol. 51, 7300504, Mar. 2015.

[7] F. Fiorillo, Characterization and Measurement of Magnetic Materials, Academic Press, 2005.

[8] F. Ikeda, A. Kobayashi and K. Fujiwara "2-D magnetic property oriented to magnetic field analysis," Joint Tech. Meeting Static Apparatus Rotating Machinery, IEE Japan, SA-04-65/RM-04-89, 2004.

[9] S. Higuchi, T. Nakao, Y. Takahashi, T. Tokumasu, K. Fujiwara, and Y. Ishihara, "Modeling of two-dimensional magnetic properties based on one-dimensional magnetic measurements," IEEE Trans. Magn., vol.48, pp. 3486-3489, Nov. 2012.

[10] T. Matsuo and M. Shimasaki, "An identification method of play model with input-dependent shape function," IEEE Trans. Magn., vol.41, pp. 3112-3114, Oct. 2005. 